\title{
Intravenous Patient-Controlled Morphine and Intrathecal Morphine Analgesia After Tibial Fracture Surgery Under Spinal Anesthesia
}

\author{
Hamid Kayalha, ${ }^{1}$ Alireza Jahangirifard, ${ }^{2}$ Abolghasem Ahmadvand, ${ }^{1}$ and Siamak Yaghoubi ${ }^{1,3, *}$ \\ ${ }_{1}^{1}$ Velayat Clinical Research Development Unit, Qazvin University of Medical Sciences, Qazvin, IR Iran \\ ${ }^{2}$ Tracheal Diseases Research Center, National Research Institute of Tuberculosis and Lung Diseases (NRITLD), Shahid Beheshti University of Medical Sciences, Tehran, IR Iran \\ ${ }^{3}$ Department of Surgery, Shahid Rajaei Hospital, Qazvin University of Medical Sciences, Qazvin, IR Iran \\ *Corresponding author: Siamak Yaghoubi, Department of Surgery, Shahid Rajaei Hospital, Qazvin University of Medical Sciences, Qazvin, IR Iran. Tel: +98-2833760620669, \\ Fax:+98-2833790611, E-mail: drsiamakyaghoubi@gmail.com
}

Received: March 1, 2015; Accepted: May 10, 2015

\begin{abstract}
Background: Physiological responses to pain and trauma have negative and dangerous effects on all organs.
Objectives:This study aimed to compare intravenous patient-controlled analgesia(PCA)and intrathecal morphine in patients undergoing tibial fracture surgery under spinal anesthesia.

Patients and Methods: This double-blind clinical trial was conducted on 80 patients undergoing tibial fracture surgery, aged 20 - 50 years and under class I and II of American Society of Anesthesiologists (ASA). They were randomly divided into two equal groups. Patients in the first group received spinal anesthesia with $3 \mathrm{~mL}$ marcaine $0.5 \%$ and $2 \mu \mathrm{g} / \mathrm{kg}(1 \mathrm{~mL})$ morphine. Patients in the second group received the same, but instead of morphine we used $1 \mathrm{~mL}$ of distilled water. In the second group, immediately after the spinal anesthesia, $1 \mathrm{mg} /$ hour morphine PCA pump was connected to the patients. In both groups, the following variables were assessed every hour for 12 hours by a third party (a trained anesthesiologist assistant): pain through visual analog scale, nausea, vomiting, pruritus, respiratory complications, mean arterial pressure, and heart rate. Data were collected using a questionnaire and then analyzed by Student's t-test, repeated measures analysis of variance (ANOVA) and chi-squared tests in SPSS software.

Results: The ANOVA test showed that there was no meaningful difference in pain between the two groups within the 12 hours after the surgery, based on visual analogue scale (VAS) (despite a slightly higher VAS in group one) $(\mathrm{P}=0.17)$. There was no meaningful difference between the patients in pruritus, nausea, vomiting and respiratory complications in the two groups.

Conclusions: Given the similar level of pain, complications, and the hemodynamic signs postoperatively in both groups, we concluded that it is better to use a single dose of intrathecal morphine instead of morphine PCA pump.
\end{abstract}

Keywords: Pain; Postoperative; Morphine; Intravenous Patient-Controlled Analgesia; Intrathecal Morphine

\section{Background}

Reducing postoperative pain, especially with appropriate analgesic regimens, leads to reduced postoperative mortality. The transmission of painful stimuli to the central nervous system produces neuroendocrine stress responses and increases the local and systemic inflammatory substances. Neuroendocrine responses to pain include increased secretion of catecholamines as well as increased catabolic hormones such as cortisol, glucagon, aldosterone, rennin, angiotensin, and antidiuretic hormone and reduced anabolic hormones, all of which will lead to increased water and sodium retention, blood glucose, ketone and lactate bodies and fatty acids and ultimately leads to a hypermetabolic and catabolic state (1).

Stress response also leads to the rejection of artery bypass graft and myocardial ischemia. Stress response suppresses immunity associated with intensity of surgery trauma, which causes hyperglycemia and reduced healing of the wound. It also leads to postoperative hypercoagulability in the form of reduced level of anticoagulants and increased procoagulants and ultimately increased incidence of deep vein thrombosis and cerebral and pulmonary thromboembolism. Increased catecholamines due to uncontrolled pain will lead to increased myocardial oxygen consumption, coronary vasoconstriction, and ultimately ischemia and heart attack. Stimulation of the sympathetic system causes a delay in bowel movements and ileus. Moreover, pain causes shallow breathing and reduces coughing, followed by exacerbation of pulmonary complications $(2,3)$.

Depending on the type of surgery, chronic postoperative pain occurs in 10 - $65 \%$ of patients and lack of effective management of acute postoperative pain is an important predicting factor for it.

Preventing these changes by analgesic treatment causes beneficial short-term effects such as reducing the postoperative acute pain and accelerating the recovery as well as long-term effects such as reduced postoperative chronic pain and improved quality of life (4).

Pain control methods include intravenous (IV) and in-

Copyright (C) 2015, Shahid Beheshti University of Medical Sciences. This is an open-access article distributed under the terms of the Creative Commons AttributionNonCommercial 4.0 International License (http://creativecommons.org/licenses/by-nc/4.0/) which permits copy and redistribute the material just in noncommercial usages, provided the original work is properly cited. 
tramuscular administration of drugs, patient-controlled analgesia (PCA) and nerve blocking (5).

PCA pump can be programmed in terms of bolus doses, intervals between doses, and continuous infusion, so that an optimal dose can be determined which is not too low or not too high to cause complications. The optimal dose for a patient (who had not previously used opioids) weighing $70 \mathrm{~kg}$ is $1 \mathrm{mg} /$ hour of morphine and $40 \mu \mathrm{g} /$ hour of fentanyl, and the most common intervals between injections are 5 - 10 minutes. PCA pump does not reduce hospital stay, but reduces the need for nursing services and increases the acceptability by patients. Moreover, this method involves fewer incidences of respiratory depression (1). PCA pump may be installed both intravenously and as intra-epidural.

This technique prevents any delay in drug administration. It provides the highest flexibility in regulating drug doses and is the best match with physiological differences in patients. It is suitable for both adults and children and is preferred to intramuscular injection in controlling postoperative pain. Today, IV PCA with morphine (as the drug of choice) is considered a mainstream of postoperative analgesia (6).

Other methods include intrathecal administration of morphine. A single intrathecal administration of 0.2 - 1 mg of morphine controls pain for 24 hours. Possible respiratory depression is an important side effect of intrathecal morphine. However, such cases are more common in doses above $5 \mu \mathrm{g} / \mathrm{kg}$ weight (4).

\section{Objectives}

Considering the importance of controlling postoperative pain and increasing the use of PCA, as well as the involving problems including proper patient and nurse training and lack of cooperation between the two, heavy cost of the pump, possibility of venous catheter dislodgment, etc., it was decided to compare the analgesic effects of IV-PCA and single dose of intrathecal morphine, which is cheaper, easier, and less time-consuming.

\section{Patients and Methods}

This clinical trial was conducted in 2013 in Shahid Rajaie Hospital affiliated to Qazvin University of Medical Sciences between 20 and 70 years of age under American Society of Anesthesiologists (ASA) class I and II, undergoing tibia fracture surgery. The sample size was determined to comprise 80 patients, based on $95 \%$ confidence, $20 \%$ relative accuracy, and $20 \%$ withdrawal possibility, who were then randomly divided into equal intervention and control groups using balanced block randomization. Patients who had regularly and in large amounts used opioids, analgesics, or tranquilizers before surgery, and those with a history of pulmonary and liver problems were excluded from the study.

This study was approved by the Ethics Committee of vice-chancellor for research Qazvin University of Medi- cal Sciences and registered at the Iranian Registry of Clinical Trials IRCT2013051513340N1. After briefing the patients and obtaining the patients' consents and in the absence of contraindications for spinal anesthesia, all the patients received $0.02 \mu \mathrm{g} / \mathrm{kg}$ weight of IV midazolam, infused through $500 \mathrm{~mL}$ of normal saline. Thereafter, the first group received spinal anesthesia with $3 \mathrm{~mL}$ of marcaine $0.5 \%$ plus $1.5-2 \mu \mathrm{g} / \mathrm{kg}$ of morphine ( $1 \mathrm{~mL}$ in volume). This group also received pethidine with muscular dose of $0.5 \mu \mathrm{g} / \mathrm{kg}$ based on visual analogue scale (VAS) of 4 or higher, or when the patient prompted. The same method was used for the second group, but $1 \mathrm{~mL}$ of distilled water was added instead of morphine, and immediately after spinal anesthesia, PCA pump was installed at a dose of 1 mg/hour at 15-minute intervals.

The level of pain was assessed in both groups according to VAS every 1 - 12 hours by a third party (trained anesthesiologist assistant). Finally, VAS every hour and IV-PCA in the first 12 hours were statistically analyzed in both groups. The study was conducted in a doubleblind format, where neither the patients nor the project executer were aware of grouping of patients or drugs administered.

Data were collected according to the study checklists, including levels of anesthesia due to intrathecal and IV-PCA use of morphine, nausea and vomiting, reduced heart rate (HR) and blood pressure (BP), and respiratory complications. Statistical analysis was carried out in SPSS software using chi-squared, t-test, and repeated measures analysis of variance (ANOVA) tests.

\section{Results}

He mean age was 45 years in the PCA group and 47 years in the intrathecal group and there was no significant difference in age between the two groups $(\mathrm{P}=0.60)$. There were no significant differences between the two groups in terms of gender, weight, or height $(P>0.05)$ (Table 1$)$.

The levels of nausea and vomiting were similar in the two groups and no significant differences were found ( $P$ $=0.50$ ). This also applied to the level of pruritus in the two groups and no significant difference was found between them. The difference between the two groups in terms of respiratory problems was also insignificant ( $P$ $=0.56$ ). Furthermore, the results showed no significant difference between the two groups in use of analgesia to reduce postoperative pain $(\mathrm{P}=0.06)$ (Table 2$)$.

Assessing the hemodynamic symptoms revealed no significant differences between the two groups in mean arterial pressure or heart rate $(\mathrm{P}=0.05)$ (Table 3 ).

The level of pain in the two groups based on VAS was compared before the surgery and every hour after the surgery for 12 hours using repeated measure ANOVA test, which showed no significant difference between the two groups. Furthermore, the mean VAS in the two groups was similar at the assessment times $(P=0.17)$ (Table 4). 
Table 1. Comparison of the Two Groups in Terms of Demographic Details and Use of Analgesics a,b

\begin{tabular}{lccc}
\hline Demographic Details & $\begin{array}{c}\text { PCA } \\
\text { Group }\end{array}$ & $\begin{array}{c}\text { Intrathecal } \\
\text { Group }\end{array}$ & P Value \\
\hline Gender & $30(85)$ & $28(64)$ & 0.60 \\
\hline Male & $10(15)$ & $12(35)$ & 0.60 \\
Female & $48.8 \pm 1.47$ & $48.8 \pm 14.6$ & 1 \\
\hline Age, $\mathbf{y}$ & $76.4 \pm 8.1$ & $75.3 \pm 8.7$ & 0.63 \\
Weight, $\mathbf{~ g g}$ & $175.2 \pm 7.2$ & $172 \pm 8.1$ & 0.17 \\
\hline Height, cm & & \\
\hline a Abbreviation: PCA, patient-controlled analgesia. \\
b Data are presented as Mean \pm SD or No. (\%). \\
\end{tabular}

Table 2. Frequency Distributions of Postoperative Nausea and Vomiting, Pruritus, and Respiratory Problems in the Two Groups a,b

\begin{tabular}{lccc}
\hline Complications & PCAGroup & $\begin{array}{c}\text { Intrathecal } \\
\text { Group }\end{array}$ & P Value \\
\hline $\begin{array}{l}\text { Nausea and } \\
\text { vomiting }\end{array}$ & $4(10)$ & $6(15)$ & 0.50 \\
$\begin{array}{l}\text { Pruritus } \\
\text { Respiratory }\end{array}$ & 6 & 6 & 1 \\
problems & $0(0)$ & $1(2.5)$ & 0.56 \\
Pethidine & $6(15)$ & $11(27.5)$ & 0.12 \\
\hline
\end{tabular}

a Abbreviation: PCA, patient-controlled analgesia.

$\mathrm{b}$ Data are presented as No. (\%).

Table 3. The Comparison of Mean Arterial Pressure and Heart Rate Between the Two Groups a,b

\begin{tabular}{|c|c|c|c|}
\hline $\begin{array}{l}\text { Hemody- } \\
\text { namic Items }\end{array}$ & PCA Group & $\begin{array}{l}\text { Intrathecal } \\
\text { Group }\end{array}$ & P Value \\
\hline $\begin{array}{l}\text { Mean arte- } \\
\text { rial blood } \\
\text { pressure }\end{array}$ & $69.6 \pm 7.93$ & $70.8 \pm 10.83$ & 0.56 \\
\hline Heart rate & $80.37 \pm 6.91$ & $81.80 \pm 4.51$ & 0.74 \\
\hline
\end{tabular}

Table 4. Comparison Between the Two Groups in Terms of Same-Time Visual Analog Scale Levels a,b

\begin{tabular}{lcc}
\hline \multirow{2}{*}{ Time, $\mathbf{h}$} & \multicolumn{2}{c}{ VAS } \\
\cline { 2 - 3 } & PCA Group & Intrathecal Group \\
\hline $\mathbf{1}$ & $1 \pm 0.0$ & $1 \pm 0.0$ \\
$\mathbf{2}$ & $1 \pm 0.0$ & $1.05 \pm 0.22$ \\
$\mathbf{3}$ & $1.02 \pm 0.15$ & $1.12 \pm 0.33$ \\
$\mathbf{4}$ & $1.17 \pm 0.38$ & $1.2 \pm 0.63$ \\
$\mathbf{5}$ & $1.37 \pm 0.54$ & $1.4 \pm 0.5$ \\
$\mathbf{6}$ & $1.62 \pm 0.66$ & $1.72 \pm 0.67$ \\
$\mathbf{7}$ & $1.9 \pm 0.74$ & $2.02 \pm 1.02$ \\
$\mathbf{8}$ & $1.85 \pm 0.66$ & $2 \pm 0.32$ \\
$\mathbf{9}$ & $1.9 \pm 0.74$ & $2.02 \pm 0.57$ \\
$\mathbf{1 0}$ & $2 \pm 0.81$ & $2.22 \pm 0.61$ \\
$\mathbf{1 1}$ & $1.85 \pm 0.56$ & $2 \pm 0.32$ \\
$\mathbf{1 2}$ & $1.68 \pm 0.76$ & $1.71 \pm 0.63$ \\
\hline a Abbreviations: PCA, patient-controlled analgesia; VAS, visual analog \\
scale. \\
b Data are presented as mean \pm SD.
\end{tabular}

\section{Discussion}

The study results showed no significant differences in level of pain between the PCA and intrathecal morphine groups. Furthermore, the two groups were also similar in mean hemodynamic symptoms, pruritus, respiratory complications, nausea and vomiting and no significant difference was observed between them.

Morphine sulfate is usually the first choice in IV pump method, because it has been more extensively used and studied compared with other opioids. Although it produces an appropriate analgesia, due to its side-effects such as nausea and vomiting, respiratory depression, sedation, confusion, and urinary retention, there is a tendency to reduce the need and use an alternative. In this study, intrathecal pump was used as an alternative method (6).

In a study by Mukherjee et al. using IV pump and intrathecal pump, they were shown to reduce postoperative pain and complications in patients, and using these pumps together, compared to IV pump alone, reduced the morphine use, but no significant difference was found between the two groups in postoperative pain and complications or satisfaction with operation $(\mathrm{P}<0.05)$ (7). A study by Khalili et al. showed that pain was better controlled by IV-PCA morphine pump than by epidural pump and the overall satisfaction was higher in this method. In epidural method, pain was not fully controlled and patients experienced reduced BP on specific occasions and higher levels of pruritus (8). Greater attention should be paid to control reduced level of consciousness in IV morphine method.

In the present study, the VAS level of pain was similar in both PCA and intrathecal methods and their difference was insignificant $(P=0.17)$. However, the mean VAS in both methods over 12 hours following surgery did not fall below 3, and in some cases it was higher than 3. On the contrary, in a study by Momeni et al. investigating the level and control of pain using PCA and local methods, the VAS level of pain was less than 3 in both methods, but pain was better controlled by the PCA method (9).

Today, PCA pump is one of the most common postoperative analgesia methods, used in many hospitals countrywide. Nonetheless, prolonged exposure of patients to opioids has significant side effects. In a study by Ghahiri et al. comparing intramuscular pethidine and PCA methods for controlling postoperative pain and complications, intramuscular pethidine had better effect than PCA method $(\mathrm{P}=0.02)$ and satisfaction was also greater in the intramuscular method (10).

The incidence of nausea and vomiting due to the stimulation of chemical receptors is in the trigger region and using opioids such as morphine cause nausea by stimulating the nausea center. Local reactions to morphine injection can occur due to histamine release or preservatives; one of these reaction include itching which is greater around the nose $(11,12)$. 
Assessing postoperative complications, Ghehiri et al. reported higher levels of nausea, vomiting and pruritus in intramuscular method compared to PCA pump method. However, in a study by Trikoupi et al. the mean level of nausea and vomiting in PCA was less than that in intramuscular method (13). However, in Mukherjee's study (7), postoperative nausea and vomiting were similar in PCA and intrathecal methods, while in the present study, levels of postoperative nausea, vomiting and pruritus in PCA and intrathecal methods were not significantly different. In a study by Devys et al. postoperative complications in intrathecal method were less compared to PCA and the difference was statistically significant (14).

Opiates interfere with respiratory regulation centers such as the brain stem and the medulla oblongata. All opiates stimulating $\mu$ receptors including morphine cause dose-dependent respiratory depression through direct effect on respiratory centers in the brain stem. The effect of morphine on the respiration pattern includes reduced respiratory rate, increased apnea, delayed exhalation, and irregular breathing. Respiratory depression peaks during 15 - 30 minutes following a single analgesic dose of morphine. Morphine also reduces arterial blood pressure, which is more seen in people with reduced volume of body fluids, with subsequent bradycardia. Arterial vasodilatation is also a direct effect of morphine on vascular smooth muscles.

In the present study, severe hemodynamic symptoms were not observed in any of the patients 12 hours after the surgery; and mean arterial BP, HR and respiratory complications were similar in both groups with no significant differences between them $(\mathrm{P}=0.05)$.

Although the use of IV PCA pump is a common and acceptable method of reducing postoperative pain providing increased patient satisfaction and earlier patient discharge, it imposes huge costs due to procurement of the pump and patient and nurse training. It is also associated with problems such as venous catheter dislodgment and improper control.

The present study demonstrated similar analgesic effects and postoperative complications in both IV-PCA pump and single intrathecal dose of morphine. Therefore, the intrathecal method that has the same complications and effects, but costs less and requires less control, can be used as an alternative to IV-PCA pump.

\section{Authors' Contributions}

Conception and design, drafting of the article, final approval of the article, administrative, technical, or logistic support: Hamid Kayalha and Siamak Yaghoubi. Analysis and interpretation of the data, critical revision of the article for important intellectual content, statistical expertise, collection and assembly of data: Alireza Jahangirifard and Abolghasem Ahmadvand.

\section{Financial Disclosure}

Qazvin University of Medical Sciences.

\section{Funding/Support}

Qazvin University of Medical Sciences.

\section{References}

1. Gadsden J, Hart S, Santos AC. Post-cesarean delivery analgesia. Anesth Analg. 2005;101(5 Suppl):S62-9.

2. Grant G, Hepner D, Bass VA. Postoperative analgesia. Available from: http://utdol.com/online/content/search.do.

3. Kodali BS, Oberoi JS. Management of postoperative pain. Available from: http://utdol.com/online/content/search.do.

4. Miller RD, Eriksson LI, Fleisher L, Wiener-Kronish JP, Young WL. Miller's Anesthesia. 7th ed. Philadelphia: Cherchil Livingstone; 2010.

5. Brunton LL, Slazo J, Parker KL. Goodman and Gilman The Pharmacological Basis of Therapeutics. 11th ed. New York: Mac Graw - Hill; 2006.

6. Kara I, Apiliogullari S, Oc B, Celik JB, Duman A, Celik C, et al. The effects of intrathecal morphine on patient-controlled analgesia, morphine consumption, postoperative pain and satisfaction scores in patients undergoing gynaecological oncological surgery. J Int Med Res. 2012;40(2):666-72.

7. Mukherjee C, Koch E, Banusch J, Scholz M, Kaisers UX, Ender J. Intrathecal morphine is superior to intravenous PCA in patients undergoing minimally invasive cardiac surgery. Ann Card Anaesth. 2012;15(2):122-7.

8. Khalili G, Karimi M. Comparison of intravenous and epidural patient-controlled analgesia after knee and hiparthroplasty. J Isf Med Schol. 2012;178:7-15.

9. Momeni M, Crucitti M, De Kock M. Patient-controlled analgesia in the management of postoperative pain. Drugs. 2006;66(18):2321-37.

10. Ghahiri AA, Fereidoni F, Abdi F, Ghasemi M, Kian SO. Comparison Study on Effect of Intramuscular Petidin Injection against Intra Venues Patient Control Analgesia (PCA) after Elective Cesarean Section. J Isf Med Schol. 2011;130:33-40.

11. Borgeat A, Ekatodramis G, Schenker CA. Postoperative nausea and vomiting in regional anesthesia: a review. Anesthesiology. 2003;98(2):530-47.

12. Singelyn FJ, Ferrant T, Malisse MF, Joris D. Effects of intravenous patient-controlled analgesia with morphine, continuous epidural analgesia, and continuous femoral nerve sheath block on rehabilitation after unilateral total-hip arthroplasty. Reg Anesth Pain Med. 2005;30(5):452-7.

13. Trikoupi AP, Vassilakos D, Soultani I, Andreopoulos K, Matsi K, Metaxa V. Patient controlled analgesia versus continuous epidural analgesia and intramuscular injections. Treatment Approaches. 2008;9:197.

14. Devys JM, Mora A, Plaud B, Jayr C, Laplanche A, Raynard B, et al. Intrathecal + PCA morphine improves analgesia during the first 24 hr after major abdominal surgery compared to PCA alone. Can J Anaesth. 2003;50(4):355-61. 